\title{
Gene Editing in Life Sciences
}

\author{
Jeet Patel ${ }^{1}$ and Erin Berlew ${ }^{2}$ \\ ${ }^{1}$ Monroe Township High School, Monroe Township, NJ, USA \\ ${ }^{1}$ Horizon Academics, Washington, DC, USA
}

\section{$\underline{\text { ABSTRACT }}$}

Gene editing technologies like the CRISPR-Cas9 system have forever changed the way we analyze genetics. As we now have a way to alter genetics, we can now investigate not just how a biological mechanism works, but wonder what we could do to make it better. This paper will discuss how biotechnology and gene editing are integrated into life sciences and biology, where most applications are found. It will be divided into four sections, each addressing how gene editing technology has advanced a field in life science. Firstly, the focus will be on viral detection systems SHERLOCK (Specific High-sensitivity Enzymatic Reporter UnLOCKing) and DETECTR (DNA Endonuclease Targeting CRISPR Trans Reporter), and their importance to the current global pandemic as well as other applications. Then the attention will be diverted into the rapidly popularizing work on gene drives and attempts to drive the evolution of populations of species to benefit mankind. Next, we will discuss the link between biotechnology and species resurrection while discussing ongoing efforts and the ethics of such massive biodiversity shifts. And finally, we discuss possible solutions for the world's hunger crisis using biotechnology with a section on genetically modified crops. The intention is to properly explain the growing applications of gene editing by the end of this paper.

\section{Introduction}

Throughout the mid-20th century, humankind was set on uncovering the mysteries of life. Scientists discovered atoms, DNA, and eventually the genetic code, the code of life. They also discovered that DNA base pairs (Adenine, Cytosine, Thymine, Guanine) code for proteins using a codon system. Once we were able to understand how genes work, we set our sights high on a new frontier; how we, as members of humankind, can alter life itself. Biotechnology has provided the human race with means of accomplishing that very feat. We have been altering the phenotypic and genotypic characteristics of organisms for centuries via artificial selection of crops and animals, but the wave of biotechnological developments at the onset of the 2010s, allowed us to harness this power to edit genes and envision manmade biology beyond our wildest dreams. Whether it be through the CRISPR Cas-9 system, zinc finger nucleases, or TALENS (Transcription Activator-like Effector Nucleases), mankind can now use our understanding of the genetic code to benefit the health, development, and environment of many of Earth's species.

\section{CRISPR:}

Clustered Regularly Interspaced Palindromic Repeats (CRISPR), with the aid of a CRISPR associated protein (Cas), allows geneticists and scientists to edit the genetic material within an organism. The first evidence of CRISPR sequences in genomes was discovered in 1987 with an unusual sequence of DNA strands found in E. coli along with other types of bacteria, later archaea, with the strand dubbed CRISPR [1]. These sequences were short palindromic repeats interspersed between other genome segments. The function of this strand remained a mystery until the mid2000s due to the lack of genome sequencing technology present at the time of its discovery. The two biologists primarily responsible for the discovery of the function of the CRISPR sequence were Francisco Mojica and Christine Pourcel who independently discovered the homology between CRISPR spacer regions to plasmids and bacteriophages. 
This finding led to the conclusion that CRISPR was in fact a biological defense mechanism that would also help the organism remember viruses. It was also shown as being able to trigger the capture of viruses during invasion, showing it was a tool for developing immunity. Research into the Cas proteins determined that when paired with CRISPR, the CRISPR-Cas pairing actually assembled the immune system of many organisms. Structurally, Cas enzymes are built with both a recognition lobe and a nuclease lobe and are built in a way that allows them to function well with CRISPR sequences. The protein serves the function of providing the enzymatic machinery needed to bind to and cut DNA when working in association with CRISPR. In today's paper we will discuss three of these Cas proteins: Cas9, which is found to be useful in immunological defense and is the primary protein for cutting DNA with CRISPR, Cas12, which works to cleave DNA with the SHERLOCK sequence, and Cas13, which works to cleave RNA with the DETECTR sequence. Whenever these individual proteins are partnered with CRISPR, it was discovered that gene elements could be targeted by sequence and DNA could be cleaved at specific locations based on these sequences. Subsequently, the potential for exploitation of these systems erupted as a very real possibility of these systems was their use in slicing and making baseline edits to immune systems of multiple organisms. These theories were proven in 2007 when a CRISPR-Cas compound made successful alteration in the immune system of bacteria Streptococcus thermophilus, making it resistant to specific phage invasion [1]. Throughout years of innovation and developments made to the basic CRISPR-Cas system, scientists have been able to use this system to make mass edits in the genetic structure of organisms in a variety of species.

The process of the CRISPR-Cas9 system to edit genomes is fairly simple to grasp, but the specificity and sensitivity of the system is altered with different Cas proteins being accompanied with it. For the purposes of the description, we will discuss the CRISPR-Cas9 system, the most popular example to date. It starts with single guide RNA (sgRNA), which consists of CRISPR RNA (crRNA), a 20-basis point (bps) long sequence that specifies the sequence we plan to edit, and tracrRNA which is a binding scaffold for the Cas protein and acts as an activator for the crRNA. This sgRNA targets the location in the host genome to be edited. CRISPR recognizes the target sequence and Cas9 induces a break in the DNA. It can make single stranded breaks (SSB) or double stranded breaks (DSB) cleaving the DNA at the target location. Finally, a repair template which stretches between 40-90 base pairs on each side, specifies the sequence to be inserted at the DSB or SSB, repairing the broken DNA via homology directed repair (HDR).

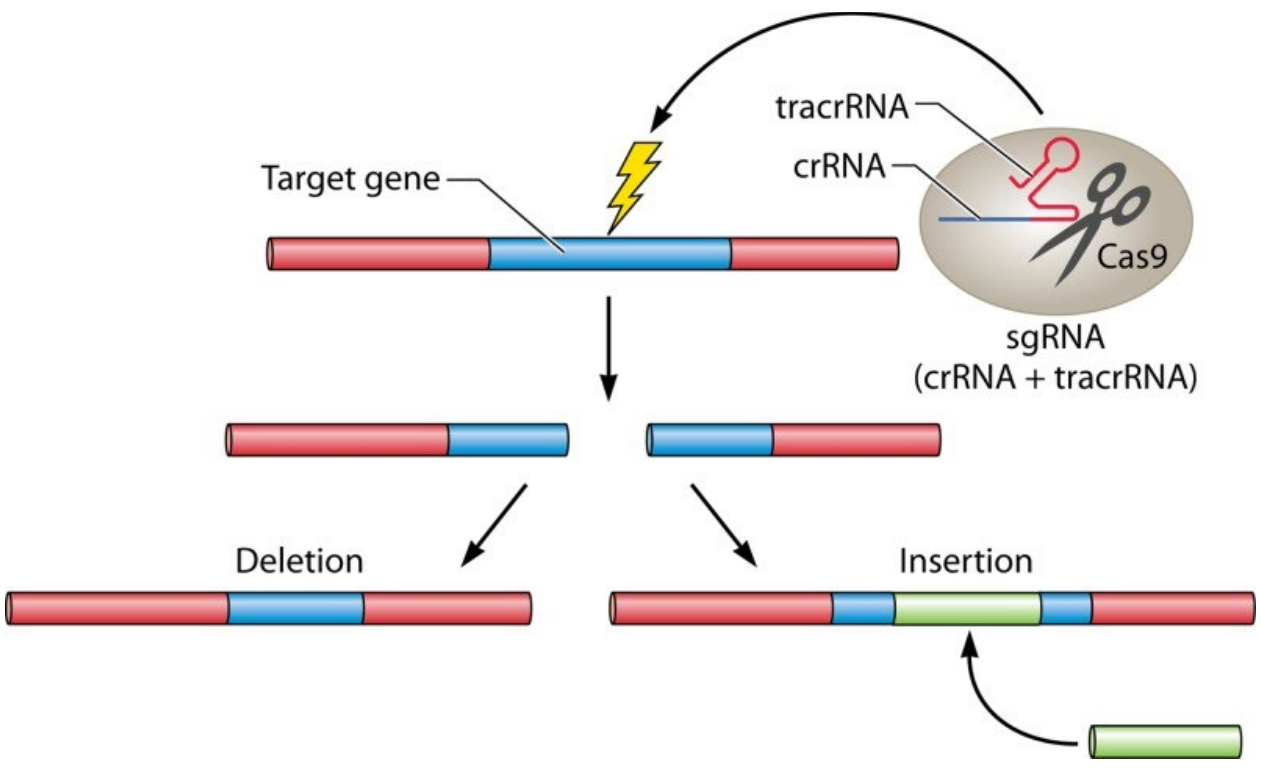

Figure 1

(Reproduced from Ishino, et al. 2018) [1] 
Genome editing by CRISPR-Cas9. The double stranded DNA is cleaved by the protein complex at the specific genome to be edited. The presence of crRNA and tracrRNA, linked together as sgRNA transports the Cas9 nuclease to the target site to be cleaved. Once the cleavage has occurred, a simple insertion and deletion mutation can completely alter the function of the gene.

There are limitations to where Cas9 can cleave DNA with CRISPR [2]. Cas proteins can only cut at a protein specific cluster of nucleotides called prospector adjacent motif (PAM). The PAM site of Cas9 specifically is NGG, or any nucleotide followed by two guanines, which is a PAM site which is quite easily found within most genomes at around every 8-16 nucleotides. The frequent occurrence of the PAM site is what makes Cas9 one of the most popular CRISPR associated proteins as it is barely limited by the structure of the genome [3].

Unfortunately, there can be some downsides to CRISPR gene editing. CRISPR has the possibility to create off target effects on the genome being edited. For example, if there is a mistake in where the DSB is introduced, the gene could become mutated, increasing the possibility for cellular apoptosis or uncontrolled cell division [3]. Also, there is a possibility of a mistake occurring during HDR which can replace a faulty gene with its homologous nonfaulty gene, but can also replace the faulty gene with another faulty gene rather than the homologous beneficial gene that rids the sequence of its illness. In this instance, money, time, and resources could be lost with the preparation of the edit resulting in no change in the final product.

We will be observing how these principles are applied to the CRISPR-Cas9 system with occasional mentions of related gene editing technologies like TALENS and Zinc Finger Nucleases to environmental conservation, to agriculture, and even to public health. CRISPR technologies have been heavily applied in this sector of science, focusing on how to further benefit the world with the use of biotechnology. We will discuss the direct applications of these gene editing technologies in life sciences along with touching on some ongoing and past examples of the use of the CRISPR-Cas system.

\section{Viral Detection Systems}

\section{Overview of Viruses:}

Before discussing the complex detection systems that exist for identifying viruses, we first need to discuss the makeup of these viruses. Viruses can either be composed of DNA or RNA, with the primary difference being that DNA viruses are more often double stranded strains while RNA viruses are primarily single stranded. These double and single stranded viral DNA genomes can be linear or circular, in the case of human papillomavirus (HPV). Some examples of DNA viruses known today are the herpes virus, poxvirus, papilloma virus, and the adenovirus. Examples of the RNA viruses are the AIDS virus, coronavirus, influenza virus, rota virus, and polio virus. Viruses can take on multiple shapes as well. These include tube-like, polyhedral, rounded, and phage viruses that all have unique methods of delivery into an organism. Diagnosing these viruses before symptoms start is often crucial to maximizing chances of survival. There are several methods of detecting a virus in a human patient. We will focus on those based on gene editing technology, namely SHERLOCK and DETECTR.

\section{Metrics for Detection:}

During a time where viral detection is becoming increasingly relevant, two major tools for detecting viruses are SHERLOCK and DETECTR based on the CRISPR gene editing system. These systems can detect either RNA or DNA viruses, but when programmed properly, both can be adapted to detect either type of virus. There are two key metrics for viral detection: the sensitivity and specificity of the system [4]. Sensitivity is a system's ability to detect extremely low amounts of viral nucleic acid (around attomolar / 10^18). Many viruses encode for very limited proteins 
and occasionally there will not be enough genetic material for the system to detect. Due to this, the goal of detection systems is to identify early-stage infections when relatively few copies of the viral genome are present. With the sensitivity of either SHERLOCK or DETECTR, the virus can often be detected before the symptoms set in. Specificity is the ability of the detection systems to identify the sequence of interest without false positive results. When the specificity of the system is low, the detection process will come out with high rates of more false positives - we'd expect false negatives if the specificity was too high.

\section{Detection Process:}

SHERLOCK or Specific High-sensitivity Enzymatic Reporter UnLOCKing is a newly emerging viral detection system which detects RNA viruses using the Cas13 enzyme in conjunction with CRISPR. On the other hand, DETECTR, DNA Endonuclease Targeting CRISPR Trans Reporter, uses the Cas12 protein rather than Cas13, and is responsible for detecting DNA viruses. SHERLOCK and DETECTR both undergo the same general process to achieve the ultimate goal of detection [5]. Both proteins used in these systems, Cas12 and Cas 13, both cleave nucleic acids promiscuously meaning that they will continuously cleave DNA or RNA upon reaching the virus and will be indiscriminate of the sequences of nucleic acids. Note that this happens following an initial sequence recognition event. When the proteins recognize their target sequence, they cleave away the target sequence following the PAM and then surrounding nucleic acid molecules [5].

However, by itself, there is no way to tell whether cleavage has occurred in the cell. To counteract this issue, researchers couple the cleavage system with fluorescent nucleotide probes which allows us to detect a cut and therefore a viral infection. The probe contains a fluorescent group and quencher on opposite ends. This quencher absorbs fluorescence from the other end of the probe, preventing fluorescence from being detected, but when Cas reaches its target sight and begins cutting, the probe itself is cut due to promiscuity, removing the quencher and allowing the fluorescent protein to begin exhibiting fluorescence and marking the spot where the cleavage first occurs [5]. Scientists conducting the experiment will be able to observe fluorescence and determine the presence of viral genetic material. The amount of virus present can be calculated from observed fluorescence using Beer's law.

a
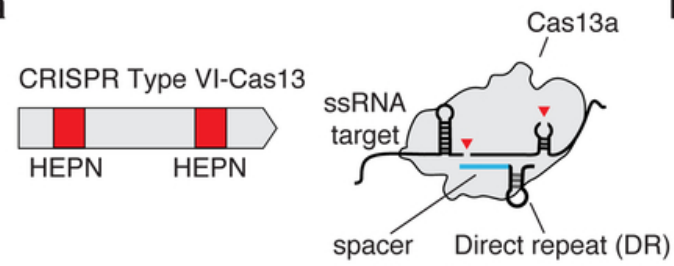

C

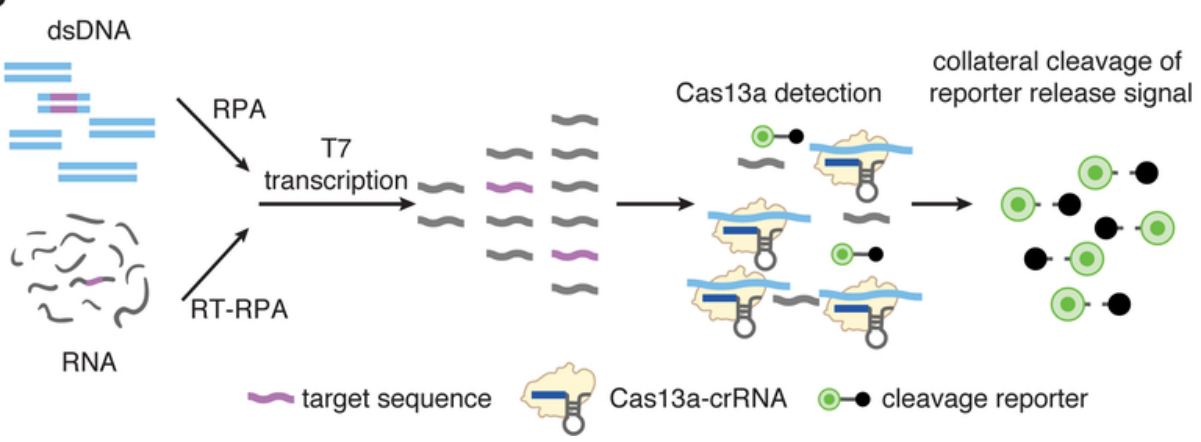

RNA sensor

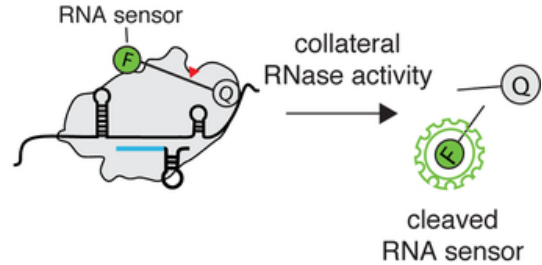

Figure 2

\section{Cas 13 complex and collateral activity}

The Cas13 protein complex consists of direct repeat sequence, spacer sequence, and ssRNA target with RNA cleavage being mediated by higher eukaryotic and prokaryotic nuclease domains (HEPN). When binding to a complementary 
target RNA, the Cas13 complex is activated triggering the cleavage of a fluorescently labeled RNA sensor which is quenched, without color, when attached to the complex and exhibits fluorescence when being cleaved. In order to use this Cas13 or SHERLOCK assay to detect, the DNA or RNA must be preamplified to the target amount and then converted to an RNA state for the Cas 13 to activate. The same process occurs with Cas 12 or DETECTR with the exception of the transcription step. (Reproduced from Kellner, et al. 2019) [5]

When conducted in a lab setting the processes of SHERLOCK and DETECTR are as follows. First, reagent preparation and second sample extraction, both of which are optional and are meant to purify the enzyme LwaCas13a, which cleaves RNA, but can be adjusted to also purify Cas13b or Cas12 in preparation for the next steps. The next two steps are third, isothermal target nucleic acid pre-amplification and CRISPR-Cas13 or Cas12 nucleic acid detection. The steps are part of the preamplification process which cleaves a nucleic acid reporter and generates a detectable signal [5]. The goal is to increase the amount of starting genetic material so it's easier to detect in our sample and to do so, we must start by revisiting the central dogma of biology. The central dogma is a flow of information that encapsulates the growth and creation of life from DNA strands to the result of a protein. We can use the central dogma to increase the amount of starting RNA or DNA. From the perspective of SHERLOCK, we begin with a few molecules of RNA where the cleavage is too difficult to detect, therefore we must attempt to increase the presence of these molecules. To do this, we use the process of reverse transcription to convert these RNA molecules back to DNA before replicating these strands. At this point we have increased the presence of genetic material, but we need to transcribe this DNA back to RNA to increase the amount of starting material, which increases the amount of Cas-mediated cleavage and the fluorescence output signal. DETECTR goes through a much simpler process, as there is no transcription needed. For the signal to increase we must simply replicate the DNA strand in a process similar to the polymerase chain reaction [6]. These pathways open the possibilities for the viral detection systems to distinguish viral DNA in both RNA and DNA viruses as transcription and reverse transcription would allow for the change of form with the viral genome present. This way of detecting both DNA and RNA viruses is done primarily in SHERLOCK as it is generally a more effective system due to its low cost, speed, wider target range, flexibility, and specificity in comparison to the DETECTR method.

\section{Applications}

Both DETECTR and SHERLOCK systems are currently in use for detecting a multitude of viruses. SHERLOCK can be used for any situation that requires sensitive detection of a DNA or RNA sequence. SHERLOCK has been used for the detection of bacterial and viral infectious diseases. It has been able to distinguish measures of the smallest values to identify the exact amount of disease in a system and have been able to detect Zika and Dengue virus directly from patient samples. SHERLOCK can distinguish between Zika strains from Honduras, the Dominican Republic, and the United States, even in regions that differ only by a single nucleotide. SHERLOCK has proven to be life-saving when applied to genotyping patient samples and detection of cancer-associated mutations from circulating cell-free DNA, even in samples where the target was present at an abundance of $0.1 \%$ compared to background [5]. There has been preliminary success in COVID-19 colorimetric diagnostic, but is not yet approved for clinical use.

DETECTR DNA detection systems also have a wide variety of applications in society. The most applicable is its ability to test for COVID-19. While like SHERLOCK, it is not FDA-approved, DETECTR looks very promising as a possible upcoming test. This process uses a lateral flow strip with a FAM Biotin Reporter to determine the virus' presence. An ssDNA probe of FAM and biotin is placed on the strip, with the next step determining the result. If the probe is uncut, the biotin will stick to the negative band with FAM still attached, giving a negative test result. If the probe is in fact cut in response to Cas recognition of a COVID-specific sequence, biotin sticks to the negative band while FAM sticks to the positive band, showing a positive test result, displayed by the presence of a line at the FAM attachment location which is lacking in negative tests [7]. DETECTR can also be used to determine the presence of Merkel cell carcinoma, a form of skin cancer [8]. 


\section{Conclusion}

The SHERLOCK and DETECTR viral detection systems may seem irrelevant to the field of organismal biology or the rest of this paper; however, detection systems have an enormous impact on human life as a whole. Detecting the presence of a viral compound early, before symptoms set in, could be the difference between life or death. While it may not be impactful to all the world's species, biology is not limited to wild animals, it includes studies of the human race, which gains tremendous benefits from biotechnology.

\section{Gene Drives}

\section{Definition of Gene Drive:}

The gene drive is a method of genetic engineering that's purpose is to increase the chances for a specific trait to be passed down through generations. However, gene drives are not primarily used as aids to natural selection; in fact, they are most often used for the exact opposite. Natural selection is the driving force behind evolution, the idea that organisms that are better suited for their environment have the highest chances of surviving and passing down genes. Gene drives often break the rules of natural selection, allowing genetically neutral disadvantageous traits to spread through a population rather than be eliminated. A handful of mechanisms exist to infuse the gene drives into the genome to create long lasting suppressive effects on a population which will be further discussed in this section.

\section{Selfish Genetic Elements:}

Inspired by a natural phenomenon found in the genetics of many organisms, scientists were able to construct the very first gene drives. Selfish genes are genetic elements that can enhance the transmission of their own genetic sequence at the expense of another, even if the typical gene provides an advantage for the organism while the selfish gene does not. The presence of natural genetic elements that can mediate the movement of DNA within genomes, in evolution were popularized by research from American biologist George C. Williams and a book entitled The Selfish Gene by partner, English biologist Richard Dawkins. Darwin's original theory of evolution by natural selection is characterized by two entities: replicators which produce exact copies of themselves, primarily referring to genes, and vehicles which interact with the actual environment, primarily organisms. These replicators are considered to be faithful while vehicles are considered to be temporary. Throughout his book, Dawkins proposes the theory of the selfish gene being used to catalyze evolution rather than altruistic behaviors and natural selection. The theory of the selfish gene was part of a much larger theory that the gene itself is the central unit of evolutionary selection [9]. When this portion of the theory is joined with the work of Leslie Orgel and Francis Crick who argue that much of the genetic material in eukaryotic genomes persists a "gene's eye view" rather than its phenotypic effects, it can be extracted that the gene IS in fact the driving force for evolution, rather than the organism.

\section{Early Gene Drives:}

Derived from the basis of this theory of genetic and selfish gene evolution came the gene drive system. While there are natural occurrences of selfish genes in organisms throughout the world, the process for gene drives is executed in labs with drives created for specific traits. In 1993 the first mechanism to innovatively edit naturally occurring selfish genes was suggested providing a start for modern gene drives, a simplification of the homing-based endonuclease drive, [10]. With the advent of the CRISPR gene editing system in the early 2010s, the buzz around this idea grew tremendously as the scientific community was finally presented with an opportunity to put this scientific dream into 
effect. Beyond using the CRISPR system, many other mechanisms to edit current selfish genes and create from scratch to create gene drives exist today.

\section{Homing Endonuclease Based Drive:}

The Homing Endonuclease Based Drive was the initial hypothesized mechanism for the creation of gene drives in Austin Burt's revolutionary paper. This process is completed by encoding a nuclease that breaks the DNA of the opposite chromosome at a specified sequence, so it has to repair itself either by non-homologous end joining (NHEJ) or microhomology end joining (MMEJ). During the repair process, the damaged chromosome will use the homing endonuclease gene (HEG) in one of two ways which will either further the invasion of the gene drive, or cause failure of the drive before the experiment can really begin. The HEG could be "selected against" and the repairing by NHEJ or MMEJ will cause the opposite chromosome to become entirely resistant against the HEG, causing drive failure, or the HEG could be used as the template for the repair to the broken DNA strand. If the HEG becomes the repair template, it will have successfully copied itself onto the opposite chromosome resulting in drive success [10,11].

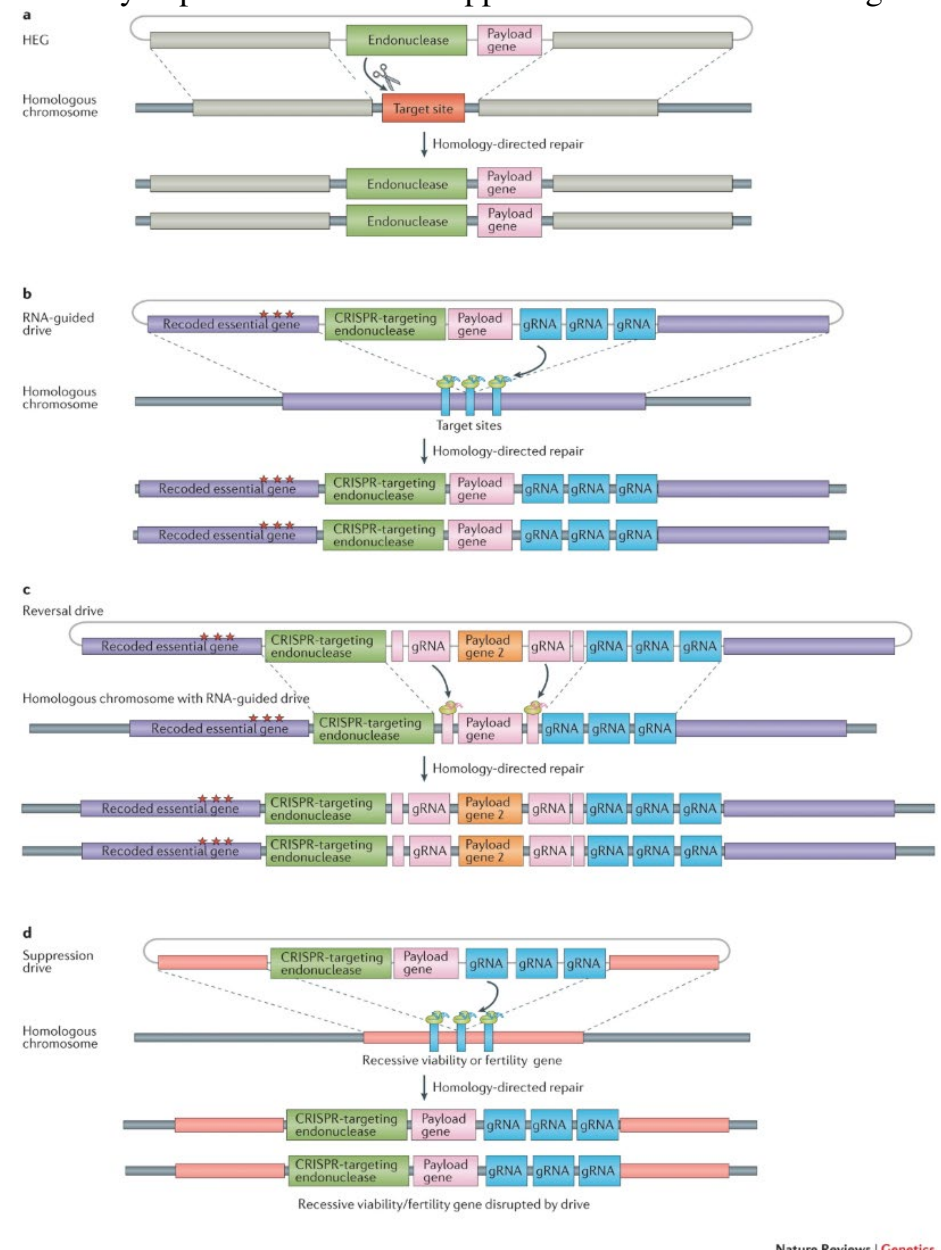

Figure 3

Homing Endonuclease genes or HEG work by first encoding a nuclease which cleaves a target site on the HEG's homologous chromosome with homology directed repair (HDR) copying the HEG onto the opposite chromosome. The second method for HEG's involve the construction of homing elements with an RNA-guided CRISPR endnuclease with guide RNAs (gRNA). To prevent resistance alleles with this process, the homing-based RNA drive would be targeted to a conserved critical gene at multiple locations with several gRNAs. The full reform would occur with HDR 
and non-homologous end joining (NHEJ). The presence of a reversal drive which encodes a gRNA targeting the previous generation drive can remove a homing based RNA guided drive from a population. Finally, the homing drives can supress populations by inducing sterility or lethality.

(Reproduced from Buchman, et al. 2016) [10]

\section{Success Probabilities:}

The chances of either of these events occurring are the same, meaning that the drive cannot be the most likely outcome. The likelihood of HEG becoming the repair template occurring increases if homology directed repair (HDR) occurs in the germline or embryonic cell, making the proportion of the offspring that receive the HEG over $50 \%$, finally breaking the rules of normal mendelian genetics and giving a more than likely chance the gene drive will continue. Due to this paper and the mechanisms ease of use, the onset of CRISPR has made it the most popular tool for creating basic gene drives. Homing Endonuclease Genes (HEG) have high success rates and have the innate ability to replicate themselves onto the opposite chromosome, ensuring the traits of the HEG gets passed on. Developing these HEGs was a monumental challenge prior to the development of the CRISPR-Cas9 system as ZFNs and TALENS were considered too bulky and repetitive to be effective when creating gene drives to suppress traits or sterilize a population. The Cas9 endonuclease was proven to have higher success in initiating a gene drive in experiments involving fruit flies, yeast, and mosquitoes. Even though HEGs are proven more often than not to be successful, limitations are still present. Oftentimes in experiments, the effectiveness of the drive seems to degrade through generations. More and more resistance alleles are developed through generations which need to be addressed when discussing improvements to synthetic HEGs. HEGs are also an irreversible gene drive, meaning that when it is incorporated into an environment it cannot be turned back to the wild type phenotype without functional resistance alleles. In some applications, this system can be effective; however, the variety of applications becomes limited as in many cases gene drives are meant to be temporary like when there is an attempt to decrease a population's numbers without causing it to go extinct as a whole in an ecosystem [10].

\section{Shredder and Sex-Linked Drives:}

The X shredder and sex-linked meiotic drive is a mechanism of gene drive that allows for the skewing of sex ratios of offspring. Many natural sex-linked selfish elements are found in the wild being skewed in favor of the gender carrying the meiotic driver, giving a starting place for the modern synthetic X-shredder. Naturally forming sex-linked drives are powered by a mutated version of the $\mathrm{Y}$ chromosome, called the $\mathrm{Y}$ drive, that has been indicated to break down the $\mathrm{X}$ chromosome during meiosis, producing more male offspring than female offspring. Drawn from these natural tendencies, the artificial X-shredder mechanism is propelled by an endonuclease that acts as the Y drive, destroying the $\mathrm{X}$ chromosome during spermatogenesis when used on sex chromosomes. The repair process will work similarly to that of homing endonuclease genes with NHEJ and MMEJ mending the breaks in the strand [10,11].

Uses for the $\mathrm{x}$-shredder are also being proposed on autosomal chromosomes for self-limiting population suppression, where alterations of the wild type genome allow for the spread of harmful traits and eventual suppression of the population, and for use directly on the Y-chromosome, which will lead to suppression of the female population and thus the population as a whole. Many of the same concerns that arise when HEGs are also present when discussing $\mathrm{X}$ shredder. The creation of resistance alleles that revert genes back to their original composition is a very real threat to these gene drives as evolution highly favors an equal sex ratio, which will contribute in resetting the favored sex ratio, preventing skewing until the gene drive is once again implemented into the population [10]. 


\section{Applications:}

In this section, we will discuss both physical applications of gene drives like disease control and pest removal as well as some of the methods by which they could be achieved. A prime usage for gene drive technology is to reduce the presence of disease in a disadvantageous human population, specifically diseases that are carried by animals, primarily insects, like malaria. Malaria specifically is responsible for at least one million deaths a year, (UNICEF). Gene drives engineered to take out a trait allowing transmission of malaria would be very efficient regarding this application, ensuring the population would stay the same in size and health. However, in many cases, the need for reducing disease like dengue and Zika is much higher than in other locations with a larger population of transmitters, leading to a need to bias the sex ratio or suppress the population as a whole, making infertile females that will eventually lead to a drastic decline in population.

Gene drives can also be used to eliminate or to control invasive species that have begun damaging the ecosystem. In this case, population suppression and extermination are the only plausible options as taking traits out of the phenotype of the species at hand, will not reduce ecological destruction because the population can still grow. After gene drives are implemented into a population suppression, three primary paths arise that gene drives can carry out their set out goals. The first, self-limiting population replacement is used to tame disease by introducing a gene drive allele that will prevent the species from transmitting a virus. However, after around 40 or 50 generations, the drive will stop working with the development of resistance alleles and eventually the population will revert back to its original code, which means we need to reintroduce the drive. The second mechanism is called global population suppression which is used for both purposes of the gene drive, disease eradication and invasive species control. This path propagates a gene through a population causing the population to crash. Specifically, it ensures that most of the offspring become males and those that become female are not fertile. Therefore, without being able to reproduce eventually the entire population will become extinct, preventing disease from spreading and getting rid of the environmental deficits by invasive species. The last application is called self-limiting population suppression which also can be used for both invasive species and disease eradication. It has similar effects to global population suppression; however, if used correctly, these mechanisms will allow us to temporarily suppress the population rather than extinct it and when necessary, stop releasing editing organisms into the environment which will push the gene drive out [10,].

\section{Ethics:}

While large scale implementation of gene drives is an intriguing prospect, many ethical questions posed by the scientific community serve as massive hurdles to the widespread use of gene drives.

\section{Moral Ethics:}

The first major concern, as with any bio-technological advancement, is what is called virtue ethics, a simple question of whether the technology breaks a personal moral high ground. It requires asking if scientists are playing God and nature or if this is too much power for humankind to wield. It also asks if scientists are getting too ahead of themselves and not caring about the consequences of failure. Despite these questions asked, the overall consensus is that when this kind of technology is proven to be safe and effective, the benefits to human-kind outweigh the ethical concern. Beyond the overarching ethical questions, gene drive specific questions are also posed in the masses like what would occur if an organism got out of containment and should scientists be killing off the populations instead of finding a more harmless solution to the population [12].

\section{Technical Ethics:}

One of these questions discusses whether a technological solution is the best possible answer to the plague of disease being spread through the world. There are possibilities that scientists would have to temporarily move populations of 
humans out of where they naturally reside to ensure drive success without human harm. However, the population may be impossible or unwilling to leave the location, meaning either risk can be taken causing us to worry about infecting humans, or stopping the gene drive in its tracks. Another concern is whether technology should be used to solve every problem facing the human race. Next, there is a concern that we will be expanding to field tests far too quickly instead of continuing research. The technology is so intriguing that field tests may be carried out in rash without knowing the harm to the human population. Finally, the last main concern posed is that we may become too dependent on gene drives to solve the pandemic-like state some disenfranchised human populations live in. If other technologies are presented, they may not be used due to the popularity and dominance gene drives have in the field [12].

\section{Conclusion:}

In summary, gene drives are a tool of biotechnology focusing on making large edits to whole populations. Through the use and modification of selfish genetic elements like homing endonuclease-based drives and sex-linked meiotic drives, traits are able to be catapulted throughout the phenotype of a set population through natural reproduction. These traits then allow for the possibilities of disease and pest control, aiding in the fight to stop global issues.

\section{De-Extinction/Conservation}

\section{Need to Resurrect Species, What Species to Bring Back:}

Thanks to works of science fiction like Steven Spielberg's adaptation of Michael Crichton's Jurassic Park, the idea of de-extinction became one of the greatest links between science and the public. While most scientists laugh at the idea of resurrecting multiple species of dinosaur, the premise of de-extinction from a scientific lens gradually became more popular, especially through the TEDx-Extinction event where a plan to bring back the passenger pigeon was introduced to the world [13]. Since then, many more projects to safely reintroduce extinct species into their natural habitats have been proposed, and while large scale de-extinction is nearly impossible to get right, reviving a population by means of natural selection could be possible. Before discussing the biological processes, and ethics pertaining to de-extinction, we must first define key terms.

\section{Key Terms:}

The terms resurrection and de-extinction were used lightly for the remainder of the paper as they substitute one another. In many instances, resurrection means bringing back an exact replica, which is not always possible with deextinction. Note that for the purposes of this paper, when the term "resurrection" is used, it is because of lack of a better term that applies to the broad scope of de-extinction. Defining de-extinction is the next priority; The International Union for the Conservation of Nature (IUCN) defines the premise of de-extinction as the generation of proxies of extinct species that are functionally equivalent to the original extinct species but are not "faithful replicas." [13] The final part of this definition is contradictory to one of the methods of de-extinction, cloning, as faithful replicas or exact copies are the exact result of cloning. Due to this, the applied definition that will be used for de-extinction for the rest of this section includes both functionally equivalent or faithful replicas to be the outcome of the resurrection process. When applied to de-extinction, a proxy defines the species created that represents the organism that was to be revived. Oftentimes, we don't have complete access to the genetic makeup of extinct species, so the resurrected species will not be exact replicas, but instead very close genetic relatives that serve as proxies. Finally, the last definition necessary to understand is that of extinction as a whole. We will focus on two classifications of extinction: extinction in the wild and global extinctions which both concern the extinctions of a species in their natural habitat. 
The primary goals of modern de-extinction methods are to reverse global and in the wild extinction [13].

\section{Need to Resurrect Species:}

On the face of it, it seems as if de-extinction is a waste of resources and pop culture makes it seem as if humans always end up regretting the decision to revive species. Scientists claim that we are currently experiencing the sixth mass extinction in the history of the planet and the first human-made extinction to date. The rate at which species, known and unknown, are going extinct is alarming with the U.N. Convention of Biological Diversity determining that 150 species are going extinct each day. De-extinction mechanisms allow us a pathway to replenish the massive loss in biodiversity. While it is possible that de-extinction will never catch up with the rate of extinction, there is a hope to save species after or before they are gone [13]. This leads us to a final question before being able to get into the process of actually resurrecting a species, what species should we consider resurrecting and bringing back into the wild. The scientific community considers keystone species, species that are pivotal to the function of an ecosystem, to be the most beneficial to resurrect [13]. They contribute tremendously to an ecosystem with large biomass but don't require a significant population to make these effects. Also, there is a group of species called "umbrella species" which are not especially ecologically significant by themselves, but their habitat needs overlap with other threatened species, creating an "umbrella" of protection for all species. Modern de-extinction projects are often criticized by the scientific community for focusing on species that don't provide many ecological benefits, but are popular amongst the public [13]. However, gaining funding for resurrecting species that aren't appealing to the general population is difficult and most projects intend to create proxies that have beneficial ecological effects.

\section{Resurrection Methods and Projects:}

The methods of editing genes for de-extinction are all limited and most likely will not create faithful replicas of the species that are meant to be brought back. The most common and effective methods are cloning, back breeding, and genome editing by precise hybridization.

\section{Cloning:}

Cloning is the most popular mechanism to the public due to science culture significance as the dinosaurs in the worldfamous Jurassic Park were created by de-extinction based cloning. It is necessary to understand that without a viable DNA sample or cell from an extinct animal, no part of the de-extinction process is possible as the proxies created will be simply based on our assumptions rather than genetic evidence. During the cloning process, the nucleus of the organism to be recreated will be extracted and then placed inside an egg, without a nucleus, of the extinct organism's closest living relative [13]. The recent wave of new biotechnological advancements has allowed for preserved zygotes to undergo this type of cloning as they have been cryopreserved to be sustained for a viable offspring to be created. Currently, this mechanism is being used to save the population of the white rhinoceros through embryogenesis and cloning, allowing for a new generation of proxies to eventually take the place of the extinct rhinoceroses. The cloning mechanism is criticized for not creating faithful replicas as mitochondrial DNA and other genetic elements of different species are used to fill the gaps of preserved DNA sequences, but this argument does not hold true as that would call for the reclassification of countless hybrid species found in nature and captivity that would no longer be considered faithful replicas [13].

\section{Back-Breeding:}

Back-breeding is another method of de-extinction with its primary limitation being that it can only be achieved for extinct species where living relatives exist. Back-breeding is a version of selective or artificial breeding in which animals are bred for many generations until a near-extinct trait is brought back into the population, slowly increasing its proportion in the population until a new proxy of the extinct species is created [13]. Back breeding is the mechanism 
is also regarded as the oldest attempt of de-extinction predating the quagga effort by four decades, however naturally this process wasn't able to get far due to the lack of technology. This method of de-extinction is extremely time consuming as many organisms have a lengthy period before they become sexually mature and pregnancy can take time as well. Back-breeding is used mainly because of the straightforward procedure and the fact that it is a natural process, meaning that there is not much risk in the process. However, the use of back breeding produces proxies that are not as similar to the extinct species attempted to be brought back in comparison to other de-extinction processes [13].

\section{Precise Hybridization:}

Precise hybridization is the most applicable and arguably the most effective method of de-extinction. For hybridization the genetic sequence of the extinct species and a template species to edit the phenotype of the organism to express alleles of the extinct species. A common example used to demonstrate hybridization is that of Beth Shapiro and other molecular biologists attempting to resurrect the wooly mammoth. Between the genome sequence of the modern-day elephant and the wooly mammoth, few major differences are present. Due to natural selection, the mammoths were selected against and those that mutated to live in warmer climates were selected for. All that needs to be done to convert an Asian elephant into a proxy of a mammoth is edit a few dozen traits that will allow for the elephant to live in colder climates as associated with the wooly mammoth, including the trademark fur that is used as the main differentiator between the two species. Successful proxies can be produced and a mammoth like creature will be born from an embryo. Unfortunately, it will likely be impossible for the foreseeable future to create a species of faithful replicas of extinct organisms due to the degradation of DNA [13,14]. Modern day projects of de-extinction begin with the longest running de-extinction effort, by use of modern technology, of the quagga which is meant to restore biodiversity to the plains of Africa. Aurochs, which are the ancestors of all modern cattle breeds are also currently being studied for resurrection. These along with the modern-day projects of the aforementioned woolly mammoth and white rhinoceros are coming close to actually having a proxy of an extinct organism. However, important ethical questions are set to block their path as of right now [13].

\section{Ethics:}

De-extinction is possibly one of the most ethically concerning areas in biotechnology today. The most pressing ethical concern regarding de-extinction is the drawing away of funds from conservation into de-extinction. Many donors and organizations may be excited about the prospect of de-extinction and identify it as a much more publicly popular project in comparison to conservation. Due to this popularity, it is likely that funding will shift towards de-extinction and away from another key concern in ecological sciences, conservation. Model simulations were created to study the effects of de-extinction on government funding, but this contradicted the idea that money would be taken away from conservatory efforts. When conservation and de-extinction are funded independently it is estimated that the funding will increase for both, providing an overall benefit to the ecological community. Moral questions, as usual, are also asked for this scientific development. These include playing god by attempting to bring back extinct populations. We also have no idea what exactly will happen to these species when brought back as they could turn hostile or create environmental destruction, rather than the expected ecological prosperity [13]. To continue from that end, relocating these extinct species back into the wild could be extraordinarily difficult as habitat destruction and ecological changes since the time of their existence could cause difficulty in adjustment and a possible re-extinction [13]. 


\title{
Crop Modification
}

\author{
History of Crop Modification:
}

The idea of crop enhancement has been a biological constant since farming became the primary way of life for human populations. Growing population numbers throughout history would have likely starved if it were not for human alteration to crops. GMOs or genetically modified organisms are organisms that have had their genomes altered in some way, resulting in an altered phenotype. While we have successfully altered the genome of microbes and animals, science has found the most widespread success in the alteration of plant genome. The first recorded instances of crop modification through artificial selection, the process by which humans choose organisms to breed to yield phenotypic outcomes of their choice, were found in Southwest Asia, near the Middle East, at least 10,000 years ago [15]. Since then, the human race has been able to domesticate crops with these same techniques like wheat, barely, lentils, and most importantly corn [15].

The onset of modern genetic modification techniques occurred from the 1940s to 1960s where science finally uncovered the mystery of DNA and genetics, in the process discovering the genetic code and the vital information that genetic elements are transferable between organisms. With this information, people began conducting experiments to achieve this feat [15]. In 1973, Boyer and Cohen using different enzymes created the first GMO, a bacterium. Private labs created the first GM crops of antibiotic resistant tobacco and petunia plants. In 1990, China became the first country to commercialize GMOs. The United States aided in this catalyzation of GM crops popularity when in 1994, the Flavr Savr tomato, edited to slow down rotting, became the first FDA-approved GMO for consumption [15].

\section{Needs for GM Crops:}

The rapid popularization of GM crops did not simply arise out of a desire to experiment with new technologies, but rather as a way to cope with the globe's agricultural dilemma. The Food and Agricultural Organization projects the global population to grow to approximately 9.7 billion by 2050 and our current mechanisms of crop development and agriculture will not be able to sustain a population of that level [15]. Using GM crops could effectively counter this looming fate. Saving fields and farms from pests, a prime usage of GM crops, could prevent the loss of 290 million dollars annually due to the fact that between $20-40 \%$ of crop destruction comes at the hand of parasites. The current agricultural industry is also responsible for $80 \%$ of global deforestation and water withdrawal leading to habitat destruction and a depletion of drinking water for many nations [15].

GM crops could be a plausible solution to most of these issues. They have generated $\$ 117.6$ billion from 1996 to 2013 in global farm income alone. Along with an increase in farming revenue, GM crops increased global crop yield by $22 \%$ and reduced pesticide usage by $37 \%$. For these same statistics to be reached without the aid of GMOs, 300 million acres of land would be needed, doing more damage to the environment than good [15].

\section{Mechanism for GM Crop Creation:}

The alteration in the code of modified crops usually arises out of the three specific methods: cisgenic, transgenic, or subgenic modification. Cisgenes are introduced from a separate organism but of a similar species, transgenes are introduced from an unrelated species, and subgenic modification is responsible for either lowering gene expression from the organism's genome or abolishing it entirely. The most practical and efficient way of making edits is a combination of the CRISPR system and the use of plant parasites like Agrobacterium Tumefaciens which is most commonly used. In this process, a genome is edited by the CRISPR system by either a cisgenic, transgenic, or subgenic mutation, and is inserted into the viral plasmid of the plant parasite. When the plasmid is implanted into the crop, the viral plasmid replicates and spreads the components on its interior, in this case the edited genome. By this, we can 
fully alter the genome of a plant as it has replicated throughout its cells. Every crop requires its own unique specific procedure to yield the most benefit from modification [16].

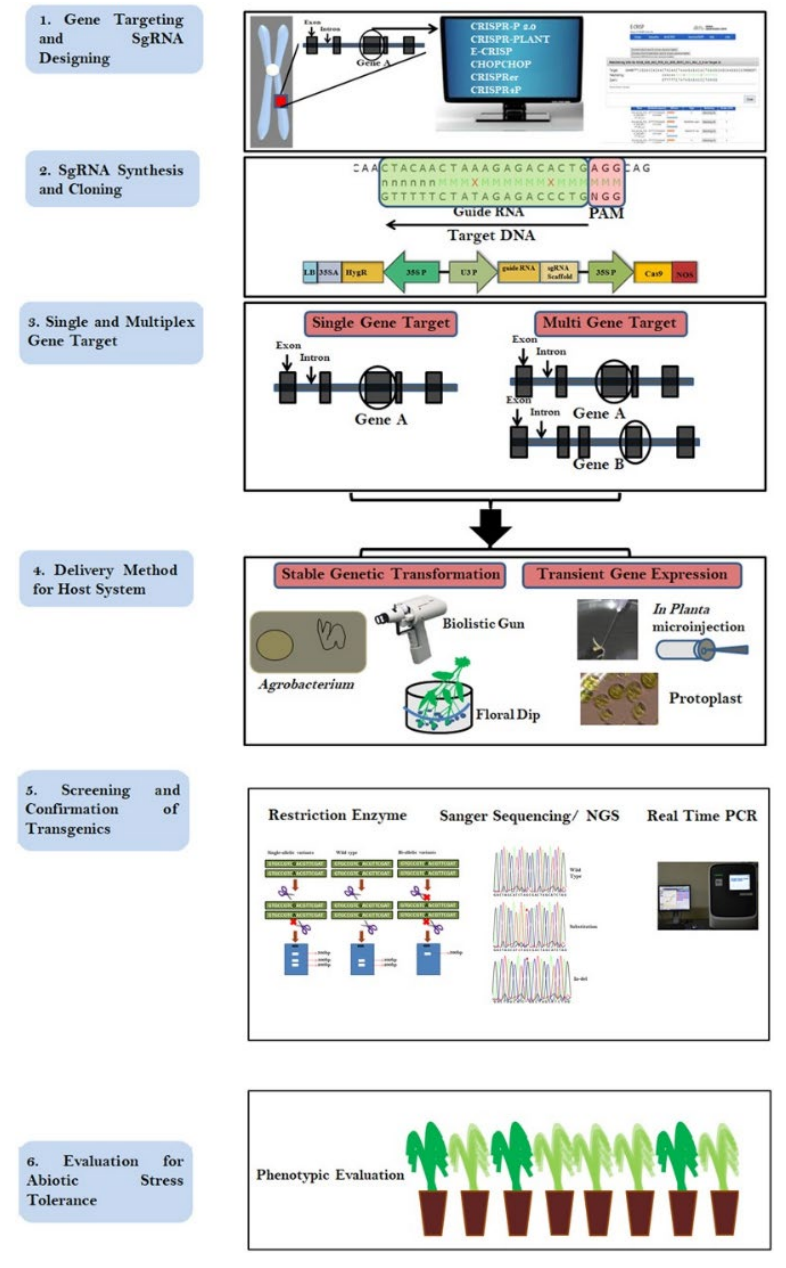

Figure 4

This figure summarizes the cultivation process of genetically modified crops. (Reproduced from Jaganathan, et al. 2018) [16]

\section{Rice:}

Rice is by almost all accounts the most reliable and steady food source on the planet due to its small size and large nutritional content. Even with this large nutritional value, increasing its nutrient content further as well as increasing its yields could do wonders for the human population. In rice crops, CRISPR has almost unlimited range to edit the genome with frequently occurring PAM sites [16]. CRISPR modification on rice crops has been completed with the three rice genes of phytoene desaturase $(O s P D S)$, betaine aldehyde dehydrogenase ( $O s B A D H 2)$ and mitogen-activated protein kinase (OSMPK2) which are responsible for controlling reactions to external stimuli. Two vectors for editing in the rice genome, pRGE3 and pRGE6, have been developed to allow RNA guided genome editing. The RNA guided editing also proved to be the most effective method as there was a much lower level of off-target effects [16].

\section{Corn/Maize:}

While the maize crop is a staple food source globally, aspects of its cultivation make it an unappealing choice to end our current and future hunger crisis, primarily due to the presence of the indigestible, anti-nutritional, pollutant found in $70 \%$ of the maize seed, phytic acid [16]. Subgenic mutation and the knockout of specific sequences in the genome 
are the primary edits when it comes to attempting to eliminate phytic acid. Genes ZmIPKIA, ZmIPK, and ZmMRP4 are involved in the synthesis of phytic acid and were knocked out in the experiment. In another experiment, the use of the maize U6 snRNA promoter allowed for the knockout of the phytoene synthase gene (PSI 1). At the conclusion of the experiment, it was discovered that every line that carried the knockout gene showed no off-target effects and was successfully edited to a more consumable form, eliminating the phytic acid present in the tested corn crops [16].

\section{Citrus:}

Citrus crops, unlike the aforementioned rice and maize, are dicots, which have two cotyledons in their seeds rather than monocots, which have one cotyledon in their seeds. While this doesn't result in a large change in procedure, the specific citrus crops are much less accessible in comparison to the other crops discussed. This is due to the fact that they require very specific growing conditions that are not found across the world, and can likely not be maintained by impoverished communities because of this. Nonetheless, the primary need for editing citrus crops like lemons and oranges is to reduce the susceptibility to citrus canker and other diseases. There is one primary gene that controls the immunity to these diseases called the lateral organ boundaries gene (CsLOB1). The removal of the effector binding element sequence from all alleles of CsLOB1 was found to be most effective with the highest resistance to citrus canker. The effectiveness of these techniques has been displayed before on the canker resistance of grapefruit and oranges [16].

\section{Soybean:}

Soybeans contain many nutritional features that are important for humans. One of the most popular examples of soybean editing using CRISPR involved the use of a single sgRNA to target two endogenous genes in the soybean genome and examine efficacy of the sgRNAs created in the root system. Targeted mutagenesis and targeted homology directed repair allowed for baseline alterations of the genetic sequence. To enhance the viral resistance of the soybean, genetic knockout experiments were carried out with pathogen virulence gene Avr4/6. Using homologous gene replacement, Avr4/6 was correctly identified and replaced with a homologous gene that would not result in decreased viral immunity [16].

\section{Present Day Applications:}

GM crops have evolved to not only be beneficial as a food source for growing populations, but a way to reap profits from farming.

\section{Indian Cotton:}

Cotton has always been considered as an important crop due to its wide variety of applications from being used as the fiber of expensive textiles or simply part of an at-home medical kit. Farmers with hundreds of acres and those with less than 15 rely on this crop for their livelihoods. This wide variety of uses makes it a vital key crop used to sustain the Indian agricultural industry. Unfortunately, like a multitude of other crops, India's cotton crops were afflicted by countless pests. The problem was continually growing out of hand, so farmers turned to genetically modified crops. In 2002, agrochemical companies Monsanto and the Maharashtra Hybrid Seeds Company (Mahyco), in a joint venture introduced Bt cotton to India. Bt cotton had the ability to produce Bacillus thuringiensis, a gene which produced toxins harmful to pests like the cotton bollworm, moths, and beetles, but harmless to other creatures like human beings. With this use of Bt crops comes more advantages like not having to worry about insecticide poisoning as the toxins don't harm humans and an increased crop yield as crops will no longer be affected by the parasites. When first introduced most farmers did not take well to Bt cotton but by $2004,46 \%$ of farmers in India were using Bt cotton [15]. Profits have increased due to Bt crops by 1877 rupees per acre and crop yield increased by 126 kilograms per acre, resulting in a $50 \%$ and $24 \%$ increase than expected without Bt cotton [15]. These percentages could even increase in the coming years with cheaper and longer lasting proxies of the crop. Bt cotton has also increased India's international economic 
status with a 22-fold increase in agro-biotechnology and a 212-fold rise in planting crops, making India the world's largest cotton exporter, overtaking China. The addition of not having to pay for insecticide, with usage cut by $25 \%$, cotton revenue in India has increased by 11.9 billion U.S dollars [15].

\section{Australian Canola:}

Canola crops in Australia provide farmers with an alternative to their normally planted crops, while still allowing them to make a profit. They also have better disease control and provide better successive growth of the cereal crops farmed afterwards. In Western Australia, between 400,000 to 800,000 acres of land are responsible for growing canola crops, which are the most popular "break crop" (alternative season crops) in the region [15]. However, despite its 440 million tonnes of yield worth a revenue of around $\$ 200$ million, canola crops are extremely susceptible to disease, especially blackleg disease, and the presence of weeds that harm the crops development, decreasing its nutritional value. Two companies aided in the relief of these issues: the aforementioned Monsanto with their product Roundup Ready and Bayer CropScience with their product, Vigor. Roundup Ready, the most commonly used of the two, contained genetic alterations making it more resistant to glyphosate. By the year 2014, GM canola's planting areas increased from 4\% to $14 \%$, throughout most of Australia [15]. Western Australia however saw an even larger boom with an increase from $0 \%$ to $21 \%$ use of GM crop areas. This dramatically increased the yield and has fueled more investment in the use of GM crops throughout the country [15].

\section{Ethics:}

While there are many ethical dilemmas created by the use of GM crops, it is important to note that despite the number, many of these concerns are slightly exaggerated by media outlets. One prominent concern is the possibility of a cancer risk emerging from GMOs due to occasional presence of higher pesticide residues linked to Hodgkins lymphoma. However, there is insufficient evidence to actually prove this statement to be true, so more data is needed to determine whether or not a risk is present. Besides the supposed risk of cancer, the gene expression and implementation of GM crops could lead to unforeseen and unexpected consequences. For example, there is a possibility of a new allergen being formed due to interactions between the new proteins formed. The alterations of the crops could create an antibiotic resistance, lowering the function of the GMO. Finally, horizontal gene transfer could provide a pathway for a deadly edit in a GM crop to enter an organism that consumed or interacted with it [17].

The last area of concern when it comes to GM crops are social issues. GM crops may be more harmful to the people it tries to help than beneficial to them. Developing countries with food shortages may be reluctant to introduce GM crops into their environment because they may jeopardize their current and future exports and may not have the funds to track and label all crops. Native cultures may be at risk as GM crops could overtake the presence of home homegrown crops. The importance of GM crops may shift the focus towards it instead of finding alternative solutions to the world's hunger crisis that would do more harm to the planet than good like developing more plantations or other possible ventures that could be identified in the future [17].

Though these crises do bring about some alarming concerns, the benefits to GM crops tremendously outweigh the differences. Our looming global food shortage needs a solution and GM crops may be the solution.

\section{Conclusion}

In summary, the vast array of genetic editing systems allows for extreme, rapid developments in the field of biology. CRISPR related proteins Cas12 and Cas13 have been found and engineered to be successful at detecting viruses. The cleavage range and combinations with fluorescent proteins have made the SHERLOCK and DETECTR viral detection systems tools for the future in regards to biomedical research and continually expanding the world's ability to protect its citizens from virus related threats. There are many prospects on the horizon for these detection systems as well. If SHERLOCK and DETECTR can be sped up to the point where it can be a commonality in medical facilities, viral 
detection can be more simplified and accurate due to the previously accurate nature of these systems $[4,5,6]$. The prospect of genetic drives being incorporated through a population was also entailed. While the animal populations experimented on will more often than not be harmed through this process, tremendous benefits appear in context of human beings. Slowly decreasing the presence of a negative genetic trait in the phenotype of a wild type species, like the ability to carry malaria, by allowing it to breed that trait through the species, can render an entire portion of the human population saved from the prospect of disease. The elimination of an entire species is also possible with the spread of gene drives, altering the gender ratio of the population to the point where it is skewed to where males make up $100 \%$ of the population, causing every organism to perish $[10,11,12]$. The notion of resurrecting lost creatures may at first glance appear insane, but with current technology, the insane is quite possible. Ongoing projects to resurrect species have been looking at procedures that could yield viable proxy organisms and have had mainly positive results in the preliminary stages. One of the most important applications for this kind of technology is its use in conservation of biodiversity along with expansion [13]. Finally, the world's most common use of biotechnology was examined, GMOs or GM crops. While the world has become accustomed to the presence of genetically modified crops, controversies are still present. Despite these controversies, the potential for GM crops to expand even farther and help reduce the issues of world hunger is present and being acted on in many places across the world $[15,16]$. Truly, the onset of genome editing has allowed for seemingly impossible discoveries and innovations for life sciences.

\section{Acknowledgments}

I would like to personally thank Ms. Erin Berlew for continuously supporting my efforts throughout the completion of this challenging project.

\section{References}

1. Ishino, Y., Krupovic, M., \& Forterre, P. (2018, March 12). History of CRISPR-Cas from Encounter with a Mysterious Repeated Sequence to Genome Editing Technology. Retrieved October 24, 2020, from https://www.ncbi.nlm.nih.gov/pmc/articles/PMC5847661/

2. Ma, Y., Zhang, L., \& Huang, X. (2014, November 07). Genome modification by CRISPR/Cas9. Retrieved October 24, 2020, from https://febs.onlinelibrary.wiley.com/doi/full/10.1111/febs.13110

3. Gupta, R., \& Musunuru, K. (2014, October). Expanding the genetic editing tool kit: ZFNs, TALENs, and CRISPR-Cas9. Retrieved October 24, 2020, from https:/www.ncbi.nlm.nih.gov/pmc/articles/PMC4191047/? ga=2.76177173.16339454.1603061089-601410891.1603061089

4. Gootenberg, J., Abudayyeh, O., Lee, J., Essletzbichler, P., Dy, A., Joung, J., . . Zhang, F. (2017, April 28). Nucleic acid detection with CRISPR-Cas13a/C2c2. Retrieved October 24, 2020, from https://www.ncbi.nlm.nih.gov/pmc/articles/PMC5526198/

5. Kellner, M., Koob, J., Gootenberg, J., Abudayyeh, O., \& Zhang, F. (2019, October). SHERLOCK: Nucleic acid detection with CRISPR nucleases. Retrieved October 24, 2020, from https://www.ncbi.nlm.nih.gov/pmc/articles/PMC6956564/ 
6. Gronowski, A. (2018, November 7). Who or What is SHERLOCK? Retrieved October 31, 2020, from https://www.ncbi.nlm.nih.gov/pmc/articles/PMC6247122/

7. Broughton, J., Deng, X., Yu, G., Fasching, C., Singh, J., Streithorst, J., . . Chiu, C. (2020, January 01). Rapid Detection of 2019 Novel Coronavirus SARS-CoV-2 Using a CRISPR-based DETECTR Lateral Flow Assay. Retrieved October 31, 2020, from https://www.medrxiv.org/content/10.1101/2020.03.06.20032334v2

8. Arora, R., Gupta, K., Vijaykumar, A., \& Krishna, S. (2019, January 01). DETECTing Merkel cell Polyomavirus in Merkel Tumours. Retrieved October 31, 2020, from https://www.biorxiv.org/con$\underline{\text { tent } / 10.1101 / 770537 \mathrm{v} 1}$

9. Ågren, J., \& Clark, A. (2018, November 15). Selfish genetic elements. Retrieved October 24, 2020, from https://www.ncbi.nlm.nih.gov/pmc/articles/PMC6237296/

10. Buchman, A., \& Akbari, O. (2016, February 15). Cheating evolution: Engineering gene drives to manipulate the fate of wild populations. Retrieved October 24, 2020, from https://www.nature.com/arti-

cles/nrg.2015.34

11. McFarlane, G., Whitelaw, C., \& Lillico, S. (2018, January 23). CRISPR-Based Gene Drives for Pest Control. Retrieved October 24, 2020, from https://www.sciencedirect.com/science/article/abs/pii/S0167779917302639

12. Callies, D. (2019, August 06). The ethical landscape of gene drive research. Retrieved October 24, 2020, from https://onlinelibrary.wiley.com/doi/abs/10.1111/bioe.12640

13. Novak, B. (2018, November 13). De-Extinction. Retrieved October 24, 2020, from https://www.mdpi.com/2073-4425/9/11/548/htm

14. Yamagata, K., Nagai, K., Miyamoto, H., Anzai, M., Kato, H., Miyamoto, K., . . Iritani, A. (2019, March 11). Signs of biological activities of 28,000-year-old mammoth nuclei in mouse oocytes visualized by livecell imaging. Retrieved October 24, 2020, from https://www.nature.com/articles/s41598-019-40546-1

15. Raman, R. (2017, October 2). The impact of Genetically Modified (GM) crops in modern agriculture: A review. Retrieved October 24, 2020, from https:/www.ncbi.nlm.nih.gov/pmc/articles/PMC5790416/

16. Jaganathan, D., Ramasamy, K., Sellamuthu, G., Jayabalan, S., \& Venkataraman, G. (2018, July 17). CRISPR for Crop Improvement: An Update Review. Retrieved October 24, 2020, from https://www.ncbi.nlm.nih.gov/pmc/articles/PMC6056666/ 
17. Hug, K. (2008, February 1). Genetically modified organisms: Do the benefits outweigh the risks? Retrieved October 24, 2020, from https://medicina.lsmuni.lt/med/0802/0802-01e.htm 